\title{
Caracterización de la comunidad vegetal en áreas de bosque nativo y plantaciones de coníferas en la Reserva Forestal Grecia (Alajuela, Costa Rica)
}

\author{
María Alejandra Maglianesi \\ Vicerrectoría de Investigación. Universidad Estatal a Distancia (UNED), Montes de Oca, San José, Costa Rica; mmaglianesi@uned.ac.cr
}

Recibido 12-IV-2010 Corregido 14-VI-2010 Aceptado 21-VI-2010

\begin{abstract}
Characterization of the plant community in native forest and exotic tree plantation at the Reserva Forestal Grecia (Alajuela, Costa Rica).

I studied plant communities in native forest and a plantation of exotic tree species (Pinus oocarpa and Cupressus lusitanica) in the Reserva Forestal Grecia (Alajuela, Costa Rica). Plant composition, richness, abundance and diversity were compared across an altitudinal gradient from 1700 to 2300 m.a.s.l. (every $100 \mathrm{~m}$ and between habitats). Fieldwork was conducted in 2008 and consisted of a total of 20 transects with a total area of 0,4 ha. The native forest had greater plant richness $\left(F_{1}=2,73\right.$; $\mathrm{P}=0,116)$ and abundance $\left(\mathrm{F}_{1}=6,51 ; \mathrm{P}=0,02\right)$ than the plantation, especially with regard to those with more exotic tree density, suggesting that light is a limiting factor for establishment of the native species. Therefore, some management measures such as the implementation of strategies for ecological restoration, to gradually replace the exotic vegetation by native species, could increase the plant diversity and then the general biodiversity in the Reserva Forestal Grecia. In addition, some plant species with particular characteristics identified in this study reveal the importance of the Reserve for conservation.
\end{abstract}

\section{KEY WORDS}

Plant richness, diversity, abundance, coniferous plantation, native vegetation, exotic plantation, Reserva Forestal Grecia, Costa Rica.

\section{RESUMEN}

Se estudió la comunidad vegetal en la Reserva Forestal Grecia (Alajuela, Costa Rica) en áreas de bosque nativo y plantaciones de especies exóticas de coníferas. Se realizaron comparaciones en la composición, riqueza, abundancia y diversidad de especies vegetales a través de un gradiente altitudinal entre los 1700 y los 2300 m.s.n.m. en bosque nativo (cada $100 \mathrm{~m}$ y entre hábitats). El trabajo de campo se realizó en el 2008 y se basó en el desarrollo de 20 transectos en total, con una superficie total de 0.4 ha. Las áreas de bosque nativo presentaron un valor promedio de riqueza $\left(F_{1}=2,73 ; P=0,116\right)$ y abundancia relativa $\left(F_{1}=6,51 ; P=0,02\right)$ mayor con respecto a las plantaciones, en particular aquellas plantaciones más densas, sugiriendo que la luz constituye un factor limitante para el establecimiento de especies autóctonas. Por lo tanto, algunas medidas de manejo como la implementación de estrategias de restauración ecológica enfocadas al reemplazo gradual de especies exóticas por nativas, podría incrementar la diversidad vegetal y con ello la biodiversidad en la Reserva. Además, se identificaron una serie de especies vegetales de características particulares, lo cual revela un alto valor de la Reserva Forestal Grecia para la conservación de estas especies.

\section{PALABRAS CLAVE}

Inventario florístico, riqueza, diversidad, abundancia, bosque nativo, plantación de coníferas, Reserva Forestal Grecia, Costa Rica.
Las plantaciones forestales han sido utilizadas en las dos últimas décadas como una solución para mitigar el problema de la deforestación, y son consideradas como una estrategia de conservación y recuperación de tierras degradadas en las regiones tropicales (Sarraihl 1984, León \& Suarez 1998, Pinillos \& Suarez 1998, SINAC 2008). Las mismas pueden brindar una serie de beneficios económicos, sociales y ecológicos (Montagnini et al. 1999), por lo que el desarrollo de estas plantaciones ha tenido buena aceptación en la práctica. Sin embargo, dichas plantaciones han recibido poca atención en la investigación, siendo escasos los estudios enfocados a determinar el papel que podrían desempeñar en la conservación de la biodiversidad.

Por otra parte, el estudio de áreas boscosas inexploradas o poco conocidas es una actividad importante, debido a que generalmente se encuentran especies que representan nuevos reportes para el país e incluso, especies nuevas que aún no han sido descritas por la ciencia. Desde el punto de vista botánico, la Reserva Forestal 
Grecia (RFG), localizada en el Área de Conservación Cordillera Volcánica Central (ACCVC), ha sido poco estudiada y la mayoría de las investigaciones se han centrado en las áreas por debajo de los 2000m de elevación, especialmente alrededor de las áreas administrativas. Por lo tanto, la mayoría de las colecciones se refieren a árboles o arbustos más o menos comunes en el país.

La RFG es de gran importancia ecológica por constituir una continuación de los límites suroccidentales del Parque Nacional Volcán Poás, de tal forma que las dos unidades de manejo en la realidad conforman una sola área protegida. Así, esta Reserva que incluye áreas de vegetación nativa, además de plantaciones de ciprés y pino, podría desempeñar un papel fundamental en la conservación de la biodiversidad, siendo de particular importancia para aquellas especies animales que realizan desplazamientos altitudinales a lo largo de su ciclo de vida.

En el presente estudio se llevó a cabo una caracterización de la comunidad vegetal en los bosques de la Reserva Forestal Grecia (RFG) a diferentes altitudes y en dos hábitats diferentes. Se analizó la composición, riqueza y diversidad florística en áreas de bosque nativo y plantación exótica de especies de coníferas, a los fines de determinar si existe la necesidad de implementar medidas de manejo que tiendan a mejorar la calidad de los hábitats para la biodiversidad. A su vez, se identificaron especies vegetales de características singulares (distribución restringida, de importancia ecológica o económica, raras o amenazadas) a fin de conocer la importancia que desempeña la RFG en la conservación de tales especies.

\section{Área de estudio}

La RFG se encuentra localizada en las faldas del Volcán Poás, a $14 \mathrm{~km}$ al norte de la ciudad de Grecia $\left(10^{\circ} 10^{\prime} \mathrm{N}\right.$, $84^{\circ} 16^{\prime}$ O). Posee una superficie de 2300 ha de bosque y un rango altitudinal que varía entre los 1600 y los $2500 \mathrm{~m}$. Fue creada en 1973 y es administrada actualmente por el Sistema Nacional de Áreas de Conservación (SINAC) a través del Área de Conservación Cordillera Volcánica Central (ACCVC) (SINAC 2008). La RFG fue establecida principalmente para la protección del recurso hídrico y además, es utilizada como área recreativa con el fin de favorecer el ecoturismo y la educación ambiental (SINAC 2008). En el año 1979, el Estado compró una finca de 40ha conocida como sector Bosque del Niño, donde actualmente se encuentra la administración de la Reserva e infraestructura para visitantes. En ese mismo año, este sector fue utilizado para la recuperación de zonas alteradas que habían sido destinadas a la ganadería y otras actividades agrícolas, utilizando 8ha para la reforestación con pino (Pinus oocarpa) y ciprés (Cupressus lusitanica) principalmente. Estas plantaciones se encuentran en un rango altitudinal entre los 1700 y los $1850 \mathrm{~m}$.

El clima de la Reserva se encuentra influido por los factores meteorológicos propios del Caribe, aunque muestra una estación seca que se extiende desde finales de noviembre hasta los primeros días de mayo. La temperatura promedio es de $16^{\circ} \mathrm{C}$ y la precipitación anual es de $3500 \mathrm{~mm}$ (SINAC 2008). Las zonas de vida representadas corresponden a Bosque muy Húmedo Montano Bajo, Bosque muy Húmedo Premontano y Bosque Pluvial Montano Bajo, predominando el primero (Holdridge 1969).

\section{METODOLOGÍA}

La fase de campo se realizó en el 2008. Con la finalidad de contar con una caracterización general de la flora, se identificaron los tipos de vegetación presentes en la Reserva, se elaboró un inventario florístico y se determinó la abundancia relativa de las especies vegetales más comunes. Además, se identificaron aquellas especies con características singulares en razón de su distribución restringida, de importancia ecológica, económica, por su rareza o grado de amenaza. Se llevaron a cabo un total de 20 transectos de 100 × 2 m (0,4ha) (ver Gentry 1988), 14 de ellos en áreas de bosque nativo (dos transectos en cada uno de los 7 rangos altitudinales ubicados cada $100 \mathrm{~m}$, desde los 1700 a los 2300 m.s.n.m) y 6 en plantaciones de especies exóticas en diferentes estados sucesionales. Con el fin de conocer el grado de interrelación entre las especies exóticas y la flora nativa, los 6 transectos correspondientes a plantación de coníferas se establecieron en 3 estados diferentes, las cuales fueron categorizadas como plantaciones degradadas, con degradación media y puras. Para el caso de las plantaciones no se consideró el factor altitud, dado que éstas se encuentran ubicadas aproximadamente a un mismo nivel altitudinal.

\section{Registro de datos}

El listado de las especies observadas se hizo mayormente mediante la identificación directa en el campo. Sólo para el caso de grupos con una taxonomía compleja y con un gran número de especies presentes en el país (e.g. Asteraceae, Bromeliaceae, Orchidaceae, Poaceae, Rubiaceae, Solanaceae, entre otras), se realizaron colecciones de herbario. Las mismas consistieron en especímenes fértiles y en casos excepcionales estériles, los cuales fueron preservados en alcohol para facilitar su posterior identificación en el herbario del Instituto Nacional de Biodiversidad (INBio) y en el Herbario del Museo Nacional. Cada una de las especies observadas fue clasificada de acuerdo a su 
familia, género y especie. Para los transectos, se marcó el mismo rumbo por medio de brújula a lo largo de una línea de 100 metros y se registraron todos los individuos arborescentes o escandentes (incluyendo lianas y bejucos) con un diámetro a la altura de pecho (DAP) superior a $2,5 \mathrm{~cm}$ (ver Boyle 1996, Cerón et al. 1997, Phillips et al. 2002), presentes a ambos lados de la línea ( $2 \mathrm{~m}$ de anchura en total).

\section{Análisis de los resultados}

Se consideró como riqueza $(\mathrm{S})$ al número de especies vegetales presentes y se determinó la abundancia relativa promedio de las 8 especies más comunes como el número de individuos presentes en los transectos por cada rango altitudinal y tipo de plantación. Se estimó la diversidad florística por medio de los índices de Shannon-Wiener, Simpson y Menhinick y se calculó la dominancia (Moreno 2001). Se realizó un análisis sobre la composición de especies a diferentes altitudes en el bosque nativo y en los dos hábitats estudiados. Para efectos prácticos, no se consideró la variable altitud en el caso de las plantaciones de coníferas. A su vez, se comparó la riqueza y abundancia relativa entre hábitats por medio de un análisis de varianza (ANDEVA) de una vía, mientras que los índices de diversidad y dominancia fueron comparados por medio de la prueba de Kruskal Wallis dado que para estos datos no se cumplieron los supuestos de las pruebas paramétricas. Para todas las pruebas se consideró un nivel de significancia del $5 \%$.

\section{RESULTADOS}

\section{Riqueza y abundancia relativa}

Se identificaron los siguientes tipos de vegetación: charral, bosque secundario, bosque primario y plantación de coníferas. Las áreas de charral no fueron contempladas en este estudio, mientras que las áreas de bosque secundario y primario fueron consideradas como bosque nativo (BN) en contraste con las plantaciones de coníferas (PC) a los fines de establecer comparaciones.

Se identificaron un total de 162 especies de árboles, arbustos, epífitas y hierbas, incluyendo algunos helechos y plantas parásitas distribuidas en 70 familias (Cuadro 1).

CUADRO 1

Número de especies vegetales por familia registradas en bosque nativo y plantación de coníferas de la Reserva Forestal Grecia (Alajuela, Costa Rica)

\begin{tabular}{|c|c|c|c|c|c|c|c|}
\hline Familia & $\mathbf{S}$ & Familia & $\mathbf{S}$ & Familia & $\mathbf{S}$ & Familia & $\mathbf{S}$ \\
\hline Acanthaceae & 1 & Clusiaceae & 2 & Meliaceae & 1 & Polypodiaceae & 1 \\
\hline Amaranthaceae & 1 & Commelinaceae & 1 & Magnoliaceae & 1 & Rhamnaceae & 2 \\
\hline Anacardiaceae & 1 & Cornaceae & 1 & Melastomataceae & 5 & Rosaceae & 1 \\
\hline Apiaceae & 1 & Cupressaceae & 1 & Meliaceae & 3 & Rubiaceae & 13 \\
\hline Apocynaceae & 2 & Cyperaceae & 1 & Monimiaceae & 1 & Rutaceae & 1 \\
\hline Araceae & 1 & Elaeocarpaceae & 1 & Moraceae & 2 & Sapindaceae & 1 \\
\hline Araliaceae & 4 & Ericaceae & 5 & Myrsinaceae & 5 & Solanaceae & 8 \\
\hline Arecaceae & 3 & Euphorbiaceae & 3 & Myrtaceae & 5 & Sapotaceae & 1 \\
\hline Asteraceae & 9 & Fabaceae & 8 & Orchidaceae & 8 & Styracaceae & 1 \\
\hline Berberidaceae & 1 & Flacourtiaceae & 2 & Papaveraceae & 1 & Symplocaceae & 1 \\
\hline Betulaceae & 1 & Gesneriaceae & 2 & Passifloraceae & 2 & Theaceae & 1 \\
\hline Bromeliaceae & 4 & Heliconiaceae & 1 & Pinaceae & 1 & Thymelaceae & 1 \\
\hline Cactaceae & 1 & Icacinaceae & 1 & Piperaceae & 4 & Tiliaceae & 1 \\
\hline Caprifoliaceae & 1 & Lamiaceae & 1 & Poaceae & 3 & Urticaceae & 1 \\
\hline Cecropiaceae & 1 & Lauraceae & 6 & Podocarpaceae & 1 & Verbenaceae & 2 \\
\hline Celastraceae & 4 & Loranthaceae & 2 & Polypodiaceae & 1 & Vitaceae & 1 \\
\hline Chloranthaceae & 1 & Lythraceae & 1 & Proteaceae & 2 & Winteraceae & 1 \\
\hline Clethraceae & 1 & Malvaceae & 1 & - & - & - & - \\
\hline
\end{tabular}


La riqueza promedio fue mayor en $\mathrm{BN}(11,4 \pm 3,9)$ con respecto a $P C(8,2 \pm 4,4)$, aunque no resultó significativa estadísticamente $\left(\mathrm{F}_{1}=2,73 ; \mathrm{P}=0,116\right)$. La riqueza promedio más baja fue de 3,5 $\pm 2,1$ especies y correspondió a la plantación pura, mientras que la riqueza promedio más alta fue de 17,5 $\pm 0,7$ especies registrada en el rango altitudinal de los 2200 m.s.n.m. (Fig. 1). Con respecto al gradiente altitudinal en el BN, no hubo diferencias significativas en la riqueza promedio de especies $\left(F_{1}=3,53 ; P=0,163\right)$.

La abundancia relativa promedio también resultó ser mayor en BN $(28,6 \pm 9,8)$ con relación a PC $(17,5 \pm$ $11,4)$, siendo esta diferencia estadísticamente significativa $\left(F_{1}=6,51 ; P=0,02\right)$. En la plantación pura se registró la abundancia promedio más baja $(3,5 \pm 2,1)$ y en el rango altitudinal de los 2200 m.s.n.m. la más alta $(44 \pm 9,9)$ (Fig. 1).

En el rango altitudinal de los 1700 m.s.n.m. las especies dominantes son el encino (Quercus seemannii, Fagaceae) y el asca (Styrax argenteus, Styracaceae), registrándose un total de 6 individuos para cada especie, seguido por el danto hediondo (Roupala glaberrima, Proteaceae) con 5 individuos. Este rango altitudinal se caracteriza por la dominancia de especies heliófitas, las cuales no llegan a alcanzar un diámetro de $2,5 \mathrm{~cm}$. Por otro lado, las palmas están prácticamente ausentes y las epífitas son muy escasas. A los 1800 m.s.n.m. predominan arbustos de cafecillo (Palicourea adusta, Rubiaceae) con 23 individuos muestreados. El danto hediondo (Roupala glaberrima, Proteaceae) es la segunda especie más común, con un total de 8 individuos. Además de las especies mencionadas, se registraron árboles de cucaracho (Billia rosea, Sapindaceae) y cacho de venado (Oreopanax xalapensis, Araliaceae).

En el rango de los 1900m.s.n.m. la especie más abundante fue el asca (Styrax argenteus, Styracaceae) con 7 individuos, seguido por el danto hediondo (Roupala glaberrima; Proteaceae), el arrayán o madroño (Vaccinium consaguineum, Ericaceae) y Viburnum costaricanum (Caprifoliaceae), con 4 individuos cada especie. La abundancia de encino (Quercus seemannii, Fagaceae) disminuyó considerablemente ya que sólo se registró un individuo. A su vez, en este rango se registró un individuo de cañuela o carrizo (Chusquea sp., Poaceae), especie que se vuelve abundante en rangos superiores. A los 2000m.s.n.m. las especies de la familia Rubiaceae fueron dominantes, siendo Rondeletia buddleoides el árbol más común, con

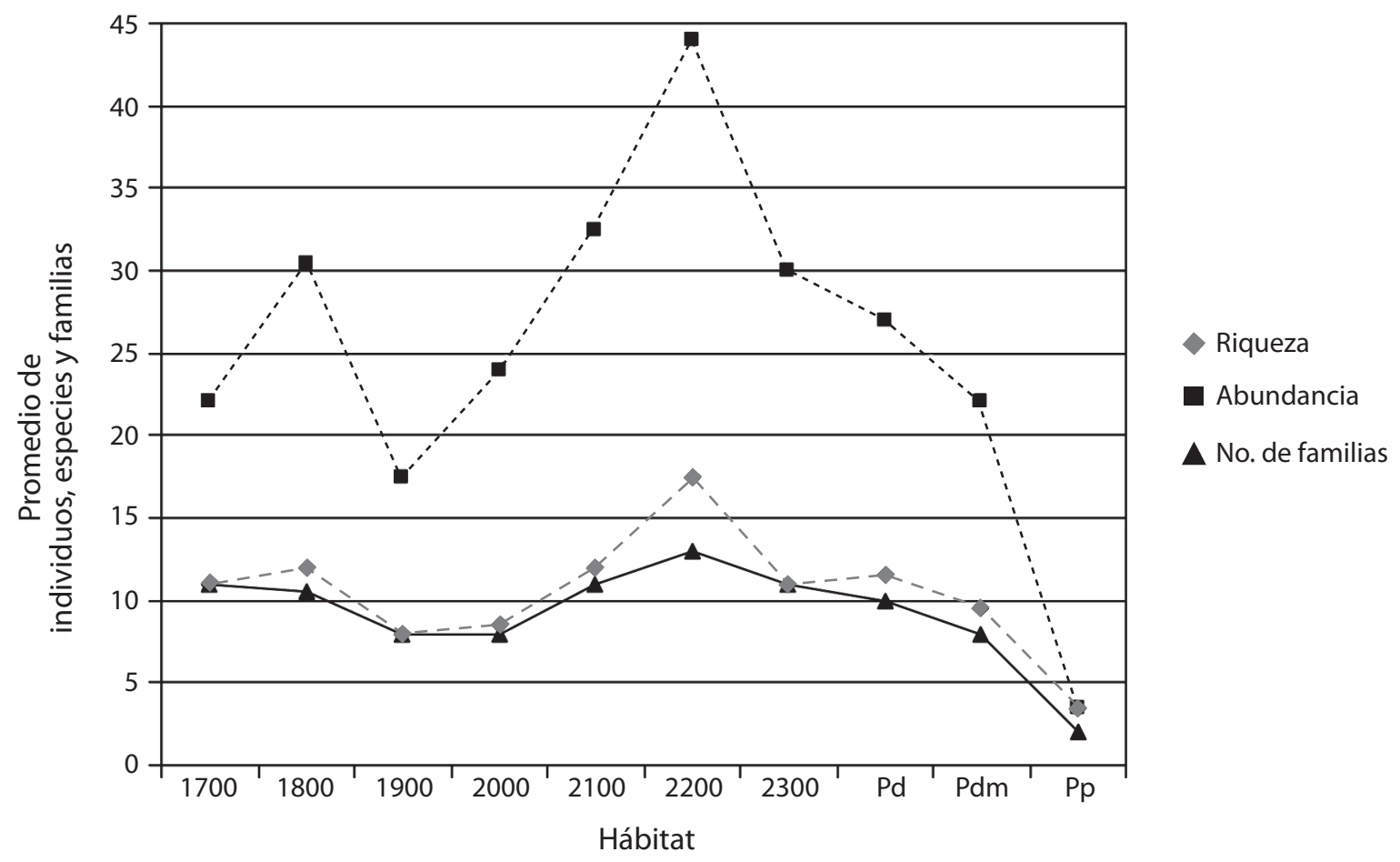

FIG. 1. Abundancia, riqueza y número de familias promedios registrados en siete rangos altitudinales (m s.n.m.) en Bosque Nativo y en tres tipos de Plantación de Coníferas en la Reserva Forestal Grecia (Alajuela, Costa Rica).

Pd: plantación degradada; Pdm: plantación con degradación media; Pp: plantación pura. 
15 individuos. A su vez, comienza la aparición de individuos de aguacatillos (Ocotea mollicela, Lauraceae). En este rango altitudinal se pueden observar individuos bien desarrollados de Celastrus vulcanicola (Celastraceae), una liana relativamente común sobre los 2000 m.s.n.m.

En el rango altitudinal correspondiente a los $2100 \mathrm{~m}$ y al igual que lo observado a los $2200 \mathrm{~m}$, la especie más común es un tipo de tucuico (Ardisia glandulosomarginata, Myrsinaceae) y se empiezan a observar árboles de cipresillo (Podocarpus oleifolius, Podocarpaceae). En estos rangos altitudinales se da la dominancia en el dosel del roble o encino (Quercus bumelioides, Fagaceae) y se observan individuos de chilenuelo (Drimyx granatensis, Winteraceae). A partir de los 2300m.s.n.m., casi en el límite superior de la Reserva, se da una dominancia de Chusquea (Poaceae) en el estrato inferior del sotobosque, llegando a convertirse en la planta más abundante, con 14 individuos. Por otra parte, los árboles de roble (Quercus bumelioides, Fagaceae) dominan el estrato superior del dosel, junto con árboles de magnolia (Magnolia poasana, Magnoliaceae) y de danto hediondo (Roupala glaberrima, Proteaceae), siendo este último uno de los árboles más comunes en la Reserva.

Con respecto a las plantaciones de coníferas, aquellas que se encuentran degradadas son las que presentaron el mayor grado de intergradación con la flora nativa. En estas plantaciones, los árboles exóticos se encuentran distanciados entre sí hasta por $30 \mathrm{~m}$, mientras que los árboles de encino (Quercus seemannii, Fagaceae) constituyen la especie más abundante, con un total de 14 individuos registrados. Las demás especies corresponden a árboles típicos de zonas alteradas como el nance macho (Clethra lanata, Clethraceae), la lengua de vaca (Miconia sp., Melastomataceae) y el cirrí (Mauria heterophylla, Anacardiaceae), los cuales estuvieron representados por 6, 5 y 4 individuos, respectivamente.

En las plantaciones con una degradación media la riqueza promedio $(9,5 \pm 4,9)$ fue más baja con relación a las plantaciones degradadas $(11,5 \pm 0,7)$, siendo la especie más común el pino (Pinus oocarpa, Pinaceae) y registrándose sólo 3 individuos de nance macho (Clethra lanata, Clethraceae) y de aguacatillo (Ocotea mollifolia, Lauraceae), además de una serie de especies que estuvieron representadas por 2 individuos o menos. En las plantaciones puras se registraron un total de 7 individuos correspondientes a un promedio de 3,5 $\pm 2,1$ especies nativas.

\section{Diversidad florística}

La diversidad florística en BN fue mayor en comparación con PC, mientras que la dominancia fue más alta en este último hábitat, aunque estas diferencias no fueron significativas estadísticamente (Fig. 2). Por otro lado, no se encontró un patrón definido de disminución de la diversidad a medida que se asciende altitudinalmente.

\section{Especies de características particulares}

A partir de los 2000m.s.n.m. se identificaron una serie de especies de características particulares, entre las cuales se encuentra el cipresillo (Podocarpus oleifolius, Podocarpaceae), la magnolia (Magnolia poasana, Magnoliaceae) y los robles o encinos (Cuadro 2). Con respecto al cipresillo y la magnolia se identificaron individuos bien desarrollados, con DAP superiores a los $70 \mathrm{~cm}$ para el caso del cipresillo. Con relación a las especies de roble, Quercus bumelioides (Fagaceae) es el árbol dominante a partir de esa elevación.

\section{CUADRO 2}

Especies de características particulares identificadas en la Reserva Forestal Grecia (Alajuela, Costa Rica)

\begin{tabular}{lll}
\hline Familia & Especie & Observaciones \\
\hline Apocynaceae & Echites turbinatus & Poblaciones reducidas \\
Berberidaceae & Mahonia paniculata & Poblaciones reducidas \\
Bromeliaceae & Werauhia ampla & Endémica \\
Bromeliaceae & Werauhia tonduziana & Endémica \\
Fagaceae & Quercus bumelioides & Poblaciones reducidas \\
Magnoliaceae & Magnolia poasana & Poblaciones reducidas \\
Orchidaceae & Encyclia mooreana & Poblaciones reducidas \\
Podocarpaceae & Podocarpus oleifolius & Poblaciones reducidas
\end{tabular}




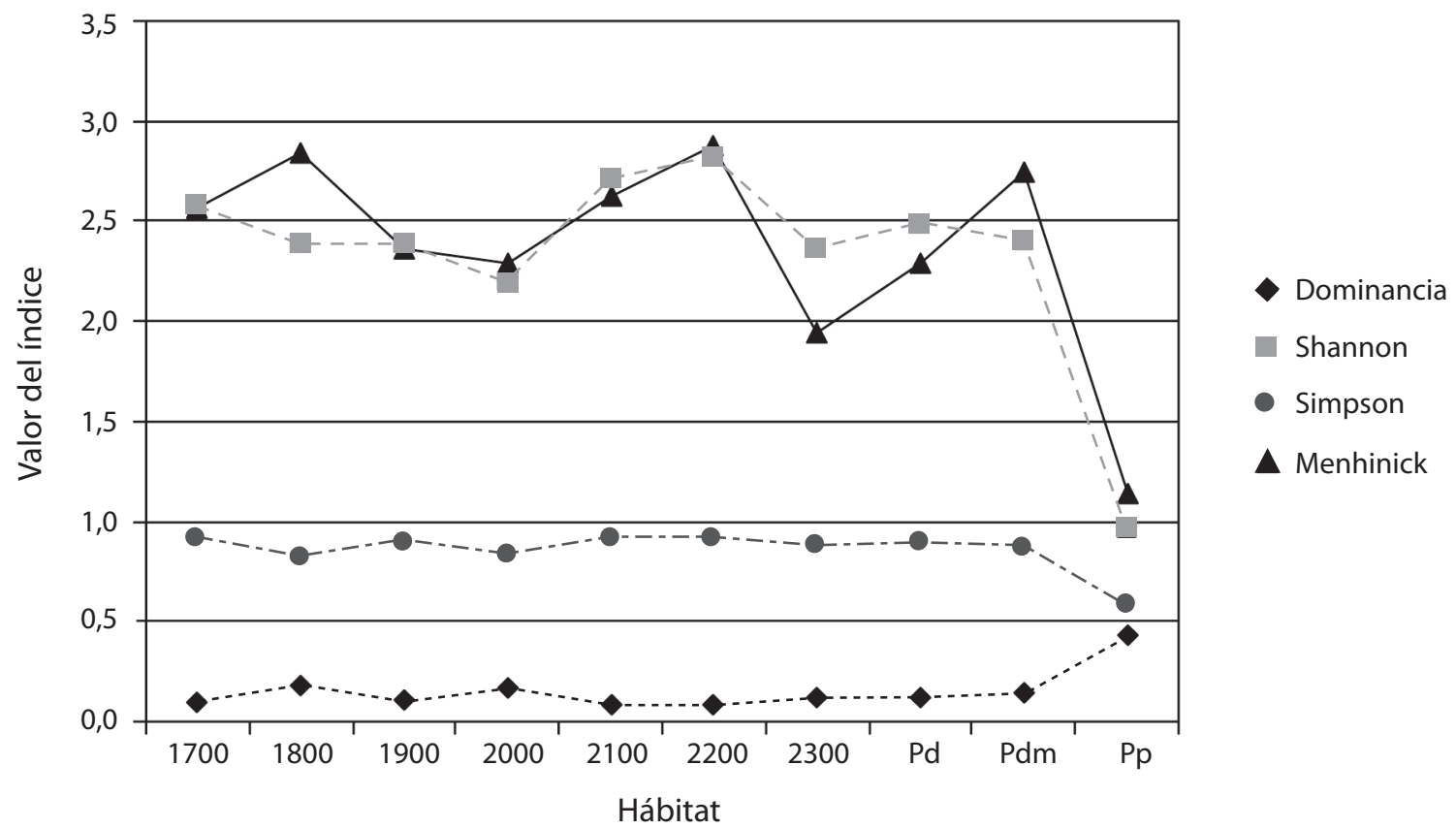

FIG. 2. Índices de dominancia y diversidad calculados en siete rangos altitudinales (m.s.n.m.) en bosque nativo y en tres tipos de plantación de coníferas en la Reserva Forestal Grecia (Alajuela, Costa Rica).

Pd: plantación degradada; Pdm: plantación con degradación media; Pp: plantación pura.

Por otra parte, se identificaron las especies Mahonia paniculata (Berberidaceae), Werauhia ampla y Werauhia tonduziana (Bromeliaceae), con poblaciones importantes para las dos últimas. Con relación a las orquídeas, las especies identificadas fueron Encyclia mooreana, al menos dos especies de Maxillaria spp. y especies de los géneros Lepanthes, Stelis y Trichosalpinx, entre otras. Otra especie singular registrada en la Reserva fue la liana Echites turbinatus (Apocynaceae).

\section{DISCUSIÓN}

\section{Riqueza, diversidad y abundancia}

Es posible analizar ciertas tendencias en cuanto a la abundancia de algunas especies que son indicadores ecológicos de las condiciones ambientales o el estado de conservación de los bosques. Así por ejemplo, los hábitats que presentan mayor alteración se encuentran a un nivel altitudinal de $1700 \mathrm{~m}$, donde están las áreas de bosque secundario y plantación de coníferas. Esto se refleja en parte por la predominancia de especies heliófitas con diámetros inferiores a los $2,5 \mathrm{~cm}$ y a la escasez de palmas y de epífitas. La predominancia de arbustos de cafecillo (Palicourea adusta) a una altitud de 1800 m.s.n.m. coincide con los resultados obtenidos para otras zonas del país, en donde los miembros de la familia Rubiaceae predominan, aunque con una composición de especies diferente (Morales et al. 2008).

Sin embargo, la falta de un patrón definido en la abundancia relativa de las especies, géneros y familias de plantas a través del gradiente altitudinal en BN de la RFG no concuerda con lo que se reporta comúnmente para otras áreas del país, tanto en la vertiente Caribe (Boyle 1996, Lieberman et al. 1996) como en la vertiente Pacífica (Kappelle \& Zamora 1995, Estrada \& Zamora 2004). Esto puede deberse a que se requiere un mayor esfuerzo de muestreo por rango altitudinal (al menos una hectárea de muestreo por cada rango) y por hábitat. Un tamaño de muestra reducido podría estar determinando también la alta dispersión en los datos encontrada para este estudio. A su vez, es importante destacar que una de las desventajas metodológicas al llevar a cabo este tipo de transectos es que no se encuentran bien representados ciertos grupos, tales como las palmas, bromelias o helechos, los cuales en algunos casos tienden a ser más sensibles ante las alteraciones extrínsecas. Por lo tanto, se requiere de estudios complementarios para poder realizar un análisis detallado de estos grupos. 
Por otra parte, la transición en la vegetación que se observa a partir de los 1900m.s.n.m. se evidencia por la presencia de plantas de cañuela o carrizo (Chusquea sp., Poaceae), una especie de bambú nativo que se vuelve dominante en el sotobosque a partir de los 2100m.s.n.m. Costa Rica tiene una alta diversidad de plantas de ese género (Morales 2003), las cuales son dominantes en el sotobosque de bosques montanos y robledales del país. El arrayán o madroño (Vaccinium consaguineum), una de las especies más abundantes a los 1900m.s.n.m., es un arbusto típicamente restringido a bosques montanos de Costa Rica y es un claro indicador de bosques nubosos muy húmedos. A su vez, una reducción considerable en la abundancia del encino (Quercus seemannii) en este rango altitudinal se debe a que esta especie es típicamente de bosques relativamente secos. Por ello, resulta abundante a alturas inferiores a los 1900m.s.n.m. donde predominan las condiciones relativamente secas características del sector oeste del Valle Central (Solano \& Villalobos 2000).

A los 2000m.s.n.m. las especies de la familia Rubiaceae dominan nuevamente, siendo Rondeletia buddleoides el árbol más común. Esta especie es facultativa ya que se puede encontrar tanto en bosques secundarios como en bosques prístinos o inalterados. De hecho, este árbol puede desarrollarse y colonizar rápidamente claros en el bosque, por lo cual su presencia no está estrictamente relacionada a una condición de bosque en particular. A su vez, la presencia de aguacatillos a este nivel altitudinal (Ocotea mollicela) concuerda con otros estudios donde se reporta que la familia Lauraceae es dominante entre los 1500 y 2000m.s.n.m. en bosques de Costa Rica (Morales et al. 2006). Por otra parte, el tucuico Ardisia glandulosomarginata, registrado en este estudio como la especie más común a los $2100 \mathrm{~m}$ de altitud, se encuentra usualmente restringido en Costa Rica a bosques nubosos con escasa o ninguna intervención sobre los 1900m.s.n.m., por lo cual la dominancia de esta especie sugiere un alto grado de madurez en los bosques estudiados a partir de esa elevación.

La dominancia en el dosel del roble o encino (Quercus bumelioides) a los 2100 y 2200m.s.n.m., posiblemente contribuya a una mayor cantidad de epífitas, las cuales se vuelven abundantes a partir de esas elevaciones, debido a que estos árboles sirven de soporte para una gran cantidad de especies epífitas. A su vez, el chilenuelo (Drimyx granatensis) es un indicador de bosques montanos, lo que indica el cambio de vegetación que se presenta a partir de los 2000m.s.n.m.

Con respecto a las plantaciones de coníferas, la falta de palmas o lianas desarrolladas, indican que estas áreas aún se encuentran en estados sucesionales tempranos (Morales et al. 2006). En las plantaciones degradadas, un mayor espaciamiento entre los árboles de coníferas posiblemente se deba a la pérdida o corta de los árboles de pino y/o ciprés, o bien a que en ciertos sectores los árboles fueron plantados a una menor densidad. En plantaciones con una degradación media, éstas se encuentran en un mejor estado de preservación, lo que implica un dosel más denso y una menor disponibilidad de luz, situación que ha influido directamente en la diversidad de especies nativas. De hecho, la presencia casi exclusiva de unos pocos individuos de encino (Quercus seemannii) con DAP considerable, posiblemente se deba a que son remanentes de la vegetación original antes del establecimiento de la plantación.

Por otra parte, las plantaciones puras carecen de algún tipo de manejo, lo que implica una alta densidad de árboles, con una mínima entrada de luz al sotobosque dificultando el establecimiento de especies autóctonas. Esto explica la baja diversidad florística y la alta dominancia que caracteriza a estas plantaciones. El patrón observado con respecto a las plantaciones sugiere que la apertura de claros podría constituir una alternativa de manejo para estas áreas con el fin de aumentar la diversidad de especies, facilitando la entrada de luz y con ello la colonización del sotobosque por especies de flora nativa. Es importante que de aplicarse dichas estrategias, sea de manera gradual, con el objetivo de reducir ciertos efectos secundarios derivados de la corta de árboles, tales como un aumento de la escorrentía, la erosión superficial, y la sedimentación de cauces de agua, entre otros.

\section{Especies de características particulares}

Debido a que la tala del cipresillo actualmente está vedada por la sobreexplotación a la cual ha sido sometido y a la presencia de individuos con diámetros considerables registrados en este estudio, la RFG constituye un área importante para proyectos relacionados con la propagación de esta especie al constituir una fuente importante de germoplasma. A su vez, cabe destacar los individuos muy desarrollados de magnolia (Magnolia poasana) observados a partir de los 2000m.s.n.m., ya que esta especie es de importancia forestal por la calidad de su madera. Finalmente, los robles o encinos, particularmente los de la especie Quercus bumelioides poseen gran importancia tanto por el valor de su madera como por el rol ecológico que desempeñan dentro del bosque.

La familia Berberidaceae es un grupo pequeño en Costa Rica, a la cual pertenece una serie de árboles y arbustos que se caracterizan por tener madera amarilla y que se han utilizado como colorantes. De la especie Mahonia paniculata registrada en este estudio se conocen muy pocas poblaciones en el país. Por otra parte, con más de 200 especies de bromelias registradas, Costa Rica tiene 
un marcado centro de diversidad de especies de la familia Bromeliaceae, la mayoría de ellas confinadas a zonas montañosas sobre los 1000m de elevación. Dos especies endémicas de las cuales se pudieron identificar poblaciones importantes en este estudio (Werauhia ampla y Werauhia tonduziana), son conocidas por pocas poblaciones localizadas en las Cordilleras Central y de Talamanca. Su presencia dentro del área de la Reserva, garantiza en parte la preservación de dichas especies a futuro.

Gran parte de las orquídeas presentes en Costa Rica son conocidas por una o dos localidades en el campo, lo cual se deriva de la falta de conocimiento y la falta de estudios sistemáticos sobre las mismas. En la RFG, se ha podido identificar una serie de especies relativamente raras, que naturalmente son escasas o cuyas poblaciones han sido mermadas por la extracción por parte de coleccionistas. Así por ejemplo, Encyclia mooreana, es una especie muy atractiva por sus flores con tonalidades verdes, lilas y blanco, y el género Maxillaria es muy buscado por coleccionistas debido a su capacidad para adaptarse con facilidad a invernaderos y por sus flores de colores vistosos. Aparte de estas orquídeas, existen otras miniaturas importantes de géneros como Lepanthes, Stelis y Trichosalpinx, entre otras, que representan un caso crítico de conservación, ya que usualmente pasan inadvertidas para los colectores no especializados y no se conoce aún el estado real de conservación de sus poblaciones en el país.

Otra especie importante en términos de conservación debido a su distribución restringida y que se encuentra presente en la Reserva es Echites turbinatus (Apocynaceae), una liana originalmente descrita para Costa Rica. Aunque se conoce actualmente que la distribución de esta especie se extiende desde Panamá a México (Morales 2005), es conocida por muy pocas poblaciones. Esta especie, al igual que las mencionadas anteriormente destaca la importancia de preservar ecosistemas con características únicas como los que se encuentran en la RFG.

En conclusión, los bosques ubicados en la RFG por debajo de los 1900m.s.n.m. presentan una diversidad relativamente baja en cuanto a especies de árboles se refiere, pues en general están sometidos a la influencia seca del sector oeste del Valle Central. Por lo tanto, se da la presencia de pocas especies que dominan el estrato superior del dosel. A partir de esa elevación se da la intergradación con bosques sometidos a una mayor disponibilidad de agua por efecto de la precipitación horizontal derivada del paso de nubes. Esto determina un cambio en la composición de la comunidad vegetal, apareciendo especies propias de áreas de mayor humedad.

Las plantaciones de coníferas poseen menor riqueza y diversidad de flora nativa, en especial las plantaciones puras, debido a que la luz representa un factor limitante para el establecimiento de especies autóctonas. Por ello, el desarrollo de estrategias de restauración ecológica enfocadas a un reemplazo gradual de especies de coníferas por nativas, podría aumentar la diversidad florística y con ello la cantidad de hábitats y biodiversidad en estas áreas. Finalmente, las especies de importancia particular registradas en este estudio, ya sea por el estado en que se encuentran sus poblaciones, por su importancia forestal, por ser de distribución restringida o por el rol ecológico que desempeñan, indican un alto valor de la RFG para la conservación de tales especies.

\section{AGRADECIMIENTOS}

Agradezco a la Vicerrectoría de Investigación de la Universidad Estatal a Distancia (UNED) por el apoyo financiero para el desarrollo de este proyecto y a Francisco Morales del Instituto Nacional de Biodiversidad (INBio) por su colaboración en el trabajo de campo. También a los funcionarios del MINAET, en particular de la Reserva Forestal Grecia por el apoyo logístico brindado y a Ligia Bermúdez por sus sugerencias para el análisis de los datos.

\section{REFERENCIAS}

Boyle, B.L. 1996. Changes on altitudinal and latitudinal gradients in neotropical montane forests. Ph.D. Dissertation. Washington University, Division of Biology and Biomedical Sciences. Missouri, St. Louis, EEUU.

Estrada, A. \& N. Zamora. 2004. Riqueza, cambios y patrones florísticos en un gradiente altitudinal en la cuenca hidrográfica del río Savegre, Costa Rica. Brenesia 61: 1-52.

Cerón, C., I. Suárez, P. Mena Valenzuela \& R. Cueva. 1997. Caracterización botánica y zoológica (mamíferos y aves terrestres) de los bosques de Santana y Arútam, Cuenca del río Pastaza, Ecuador. Ecociencia. Quito, Ecuador.

Gentry, A.H. 1988. Changes in plant community diversity and floristic composition on environmental and geographical gradients. Annals of the Missouri Botanical Garden 75: 1-34.

Holdridge, L.R 1969. Life zone ecology. Rev. Ed. San José, Costa Rica. Tropical Science Center. San José, Costa Rica.

Kappelle, M. \& N. Zamora. 1995. Changes in woody species richness along an altitudinal gradient in Talamanca montane Quercus forests, Costa Rica. p.135-148, In S.P. Churchill, H. Balslev, E. Forero \& J.L. Luteyn (eds.). Biodiversity and Conservation of Neotropical Montane Forests. Nueva York, EEUU.

León, T. \& A. Suarez. 1998. Efectos de plantaciones forestales sobre suelo y agua. Serie Técnica Nº40. CONIF, Bogotá, Colombia. 
Lieberman, D., M. Lieberman, R. Peralta \& G. Hartshorn. 1996. Tropical forest structure and composition on a largescale altitudinal gradient in Costa Rica. Journal Ecology 84: 137-152.

Montagnini, F, M. Guariguata, A. Mariscal, N. Ribeiro \& D. Shepherd. 1999. Reforestación con especies nativas para la recuperación de parcelas degradadas: experiencia en tres regiones de Latinoamérica. Primer Seminario Centroamericano. Siguatepeque, Honduras.

Morales, J.F. 2003. Poaceae. In Hammel, B. E., M. Grayum, C. Herrera \& N. Zamora (eds.) Manual de las Plantas de Costa Rica. vol. III. Monogr. Syst. Bot. Missouri Bot. Gard. 93: 1-858.

Morales, J.F. 2005. Estudios en las Apocynaceae neotropicales XIX: la familia Apocynaceae S. Str. (Apocynoideae, Rauvolfioideae) de Costa Rica. Darwiniana 43: 90-191.

Morales, J.F., N. Zamora \& A. Soto. 2006. Desarrollo de indicadores ecológicos para el monitoreo y evaluación de la integridad ecológica de los bosques, en la banda media de elevación del Parque Internacional La Amistad (PILA) y zonas de influencia. Informe Técnico elaborado para The Nature Conservancy (TNC), San José, Costa Rica.

Morales, J.F., N. Zamora \& B. Herrera. 2008. Análisis de la vegetación y proposición de posibles indicadores ecológicos en la franja altitudinal de 800-1500m s.n.m.en la vertiente pacífica del Parque Internacional La Amistad (PILA), Costa Rica. Brenesia 70.

Moreno, C.E. 2001. Métodos para medir la biodiversidad. CYTED. Zaragoza, España.

Phillips, O., J. Miller \& A. Gentry. 2002. Global Patterns of Plant Diversity Alwyn H. Gentry's Forest Transect Data Set. Systematic Botany from the Missouri Botanical Garden 89: 0-319.

Pinillos, G. \& A. Suarez. 1998. Efectos de plantaciones forestales sobre fauna y flora. Serie Técnica N41. CONIF, Bogotá, Colombia.

Sarraihl, M. 1984. Mise en valeur de l'écosystem forestier guyanais. Operation ECEREX: resumé de premiers résultats. . Bois et Forets de Tropiques 206: 13-32.

SINAC. 2008. Plan de Manejo Reserva Forestal Grecia (Borrador). Sistema Nacional de Áreas Protegidas. MINAET, San José, Costa Rica.

Solano, J. \& R. Villalobos. 2000. Regiones y subregiones climáticas de Costa Rica. Instituto Meteorológico Nacional. Gestión de Desarrollo. San José, Costa Rica. 